\title{
Editorial: Progress in Computer Gaming and Esports: Neurocognitive and Motor Perspectives
}

\author{
Adam J. Toth ${ }^{1,2}$, Cornelia Frank ${ }^{3}$, David Putrino ${ }^{4}$ and Mark J. Campbell ${ }^{1,2 *}$ \\ ${ }^{1}$ Department of Physical Education and Sport Sciences, Faculty of Education and Health Sciences, University of Limerick, \\ Limerick, Ireland, ${ }^{2}$ Lero, The Irish Software Research Centre, Limerick, Ireland, ${ }^{3}$ Institute for Sport and Movement Sciences, \\ University of Osnabrück, Osnabrück, Germany, ${ }^{4}$ Icahn School of Medicine, Mount Sinai Hospital, New York, NY, \\ United States
}

Keywords: esport science, cognition, video games (psychology), AVG, sensory-motor abilities

\section{Editorial on the Research Topic}

\section{Progress in Computer Gaming and Esports: Neurocognitive and Motor Perspectives}

This Research Topic covers the neurocognitive aspects of computer gaming and esports. Authors representing a broad spectrum of psychology and neuroscience have contributed, introducing empirical findings as well as conceptual and methodological innovations. In this Editorial we provide a thematic overview of the exciting and diverse contents of this Research Topic.

Video games have become a cultural phenomenon over the past 50 years and are now one of the most prominently chosen past times (Wagner, 2006; Hamari and Sjöblom, 2017; Lokhman et al., 2018). The use of dynamic visual displays, the demand on flexible attention allocation

OPEN ACCESS

Edited and reviewed by: Bernhard Hommel, University Hospital Carl Gustav Carus, Germany

${ }^{*}$ Correspondence: Mark J. Campbell mark.campbell@ul.ie

Specialty section: This article was submitted to Cognition,

a section of the journal

Frontiers in Psychology

Received: 26 March 2021 Accepted: 30 March 2021

Published: 22 April 2021

Citation:

Toth AJ, Frank C, Putrino D and Campbell MJ (2021) Editorial: Progress in Computer Gaming and Esports: Neurocognitive and Motor and the requirement for precise time-constrained bimanual motor control, make video games a unique medium for studying both cognition and motor control Bera et al. Over the past 20 years, neurocognitive research has demonstrated that habitual competitive video game players appear to display some superior cognitive attributes when compared to their non-video gaming counterparts (Colzato et al., 2013; Bediou et al., 2018; Kowal et al., 2018). Along with the increased recognition of esports as a sporting activity alongside traditional athletic sports, the unique cognitive skillset possessed by elite gamers has earned them the moniker of "cognitive athletes" (Campbell et al., 2018). This notion has led to an increased appetite toward understanding the cognitive benefits conferred from, and demanded by, video games.

A plethora of research has demonstrated that habitual action video gamers demonstrate superior information processing (Yuji, 1996; Dye et al., 2009; Kowal et al., 2018), attention Li et al.; Schenk et al., task switching (Colzato et al., 2010; Green et al., 2012; Toth et al., 2020), and memory abilities (Wilms et al., 2013; Waris et al., 2019), compared to non-gaming populations. Moreover, research has found these same cognitive skills can be enhanced when non-gamers engage with action video games in particular (Oei and Patterson, 2013; Clemenson and Stark, 2015). In fact, cognitive ability, in addition to past gaming experience, has even been found to predict one's ability to quickly learn new video games Smith et al. Alternatively, for some cognitive skills like response inhibition, gamers adopt a strategy that prioritizes speed over accuracy, showing no clear advantage (Kowal et al., 2018). This finding has been recently corroborated by Sousa et al., who demonstrated a decrease in inhibitory performance among participants following FPS and MOBA game play, which was larger in magnitude following FPS, compared to MOBA, play Sousa et al. Moreover, cognitive inhibition has also recently been shown not to differentiate ranking among gamers within a prominent FPS game (Colzato et al., 2013; Toth et al.). However, it is important to note that although classical tests of cognitive inhibition fail to differentiate gaming 
process, altering the context in which this skill is evaluated may also alter outcomes. For example, a gamified examination of cognitive inhibition may demonstrate performance differences among gamers, where classical standardized stroop tasks have failed to delineate differences. During F1 driving simulations for example, Eckardt et al. found that driving performance was largely tied to adaptive abilities and selective attention/inhibition ability Eckardt et al.

The recognized role of cognitive development and training in esports has extended to traditional sports, where superior executive function (EF) abilities can augment motor skill performance. For example, Beavan et al. share the importance of not only evaluating EF ability among soccer players, but also the set-up of consistent protocols and the effective communication of results to players. Traditional sport may also benefit from video games for motor learning too. In addition to the cognitive benefits that can be attained through video game play, video game environments have shown to promote motor skill practice away from the field of play. Michalski et al. demonstrate, in their recent review, that virtual environments (VE) now demonstrate a realism and flexibility that makes them potentially ideal and cost-effective for motor learning and the transfer of skill to the real world Michalski et al. Although work in this area is still sparse, early signs point to a promising role for VE to augment performance in traditional sports as well (Hülsmann et al., 2019).

In addition to the potential for video games to augment cognitive and motor abilities, they have also shown promise as a mental health tool as well (Kowal et al., 2021). Not only has this been significant in light of the recent COVID19 pandemic, but Tabacof et al. have demonstrated the critical role that video games can play in providing feelings of social connectedness among those who may have physical or mental disability Tabacof et al. In their study, they present solutions that can improve the accessibility of video games to those with spinal cord injury (SCI) and quadriplegia and how doing so significantly improves feelings of social connectedness among these individuals. Overall, this highlights the benefit that the digital nature of video gaming brings as a medium to remove barriers that may exist between individuals of varying sex, race, and physical ability.

Despite all the benefits to be had from video games, they are not without their pitfall. Their sedentary nature and the fact that users can find themselves staring at bright luminant screens for prolonged periods of time have been well cited as

\section{REFERENCES}

Bediou, B., Adams, D. M., Mayer, R. E., Tipton, E., Green, C. S., and Bavelier, D. (2018). Meta-analysis of action video game impact on perceptual, attentional, and cognitive skills. Psychol. Bullet. 144:77. doi: 10.1037/bul0000130

Campbell, M. J., Toth, A. J., Moran, A. P., Kowal, M., and Exton, C. (2018). eSports: a new window on neurocognitive expertise? Progr. Brain Res. 240, 161-174. doi: 10.1016/bs.pbr.2018.09.006

Clemenson, G. D., and Stark, C. E. (2015). Virtual environmental enrichment through video games improves hippocampal-associated memory. J. Neurosci. 35, 16116-16125. doi: 10.1523/JNEUROSCI.2580-15.2015

Colzato, L. S., van den Wildenberg, W. P., Zmigrod, S., and Hommel, B. (2013). Action video gaming and cognitive control: playing first person shooter games negative attributes (Lanningham-Foster et al., 2006; DiFranciscoDonoghue et al., 2019; Yin et al., 2020). Therefore, some are now calling for a holistic approach to esports training and video game play in general Martin-Niedecken et al. In fact, new research is now altering the way esports players train, moving away from predominant full emersion training to adopting segmented skill based variable priority training strategies, prevalent in traditional sport (Toth et al., 2021). The role of physical exercise is also emerging as critical to the health and performance longevity in esports, despite physical exertion being a fraction of what is required at the elite level in traditional sports. Moreover, in addition to the well-established physical and mental benefits, physical exercise has been shown to augment the cognitive abilities which provide top video gamer players their advantage in the first place (Toth et al., 2020).

In conclusion, despite the prevalence and popularity of video games and esports worldwide today, we are still at the frontier of esports science and are only beginning to identify the key features that make elite esports athletes unique Smithies et al. Furthermore, the cognitive benefits conferred from engaging with video games, further social and physical benefits are likely to manifest as well, in addition to the development of a better understanding of how to mitigate health and performance pitfalls associated with video game play. As we embark on a new decade, we can expect to see a surge of new research on the role video games and virtual environments play in multiple facets of our lives, and this work is welcome now as we continue to place video games at the fore front of entertainment media in our digital world.

\section{AUTHOR CONTRIBUTIONS}

AT and MC wrote the first draft. CF and DP edited the draft. All authors contributed to and signed off on the final draft.

\section{ACKNOWLEDGMENTS}

This publication has emanated from research [conducted with the financial support of/supported in part by a grant from] Science Foundation Ireland under Grant number 13/RC/2094_2. For the purpose of Open Access, the author has applied a CC BY public copyright license to any Author Accepted Manuscript version arising from this submission. is associated with improvement in working memory but not action inhibition. Psychol. Res. 77, 234-239. doi: 10.1007/s00426-012-0415-2

Colzato, L. S., Van Leeuwen, P. J., Van Den Wildenberg, W., and Hommel, B. (2010). DOOM'd to switch: superior cognitive flexibility in players of first person shooter games. Front. Psychol. 1:8. doi: 10.3389/fpsyg.201 0.00008

DiFrancisco-Donoghue, J., Balentine, J., Schmidt, G., and Zwibel, H. (2019). Managing the health of the eSport athlete: an integrated health management model. BMJ Open Sport Exer. Med. 5:e000467. doi: 10.1136/bmjsem-2018-0 00467

Dye, M. W., Green, C. S., and Bavelier, D. (2009). Increasing speed of processing with action video games. Curr. Direction. Psychol. Sci. 18, 321-326. doi: 10.1111/j.1467-8721.2009.01660.x 
Green, C. S., Sugarman, M. A., Medford, K., Klobusicky, E., and Bavelier, D. (2012). The effect of action video game experience on task-switching. Comput. Hum. Behav. 28, 984-994. doi: 10.1016/j.chb.2011.12.020

Hamari, J., and Sjöblom, M. (2017). What is eSports and why do people watch it? Internet Res. 27:85. doi: 10.1108/IntR-04-2016-0085

Hülsmann, F., Frank, C., Senna, I., Ernst, M., Schack, T., and Botsch, M. (2019). Superimposed skilled performance in a virtual mirror improves motor performance and cognitive representation of a full body motor action. Front. Robotics Artif. Intelligence 6:43. doi: 10.3389/frobt.2019.00043

Kowal, M., Conroy, E., Ramsbottom, N., Smithies, T., Toth, A. J., and Campbell, M. J. (2021). Gaming your mental health: a narrative review on mitigating depression and anxiety symptoms via commercial video games. JMIR Serious Games. doi: 10.2196/26575. [Epub ahead of print].

Kowal, M., Toth, A. J., Exton, C., and Campbell, M. J. (2018). Different cognitive abilities displayed by action video gamers and non-gamers. Comput. Hum. Behav. 88, 255-262. doi: 10.1016/j.chb.2018.07.010

Lanningham-Foster, L., Jensen, T. B., Foster, R. C., Redmond, A. B., Walker, B. A., Heinz, D., et al. (2006). Energy expenditure of sedentary screen time compared with active screen time for children. Pediatrics 118, e1831-e1835. doi: 10.1542/peds.2006-1087

Lokhman, N., Karashchuk, O., and Kornilova, O. (2018). Analysis of eSports as a commercial activity. Probl. Perspectiv. Manag. 16, 207-213. doi: 10.21511/ppm.16(1).2018.20

Oei, A. C., and Patterson, M. D. (2013). Enhancing cognition with video games: a multiple game training study. PLOS ONE 8:e58546. doi: 10.1371/journal.pone.0058546

Toth, A. J., Ramsbottom, N., Constantin, C., Milliet, A., and Campbell, M. J. (2021). The effect of expertise, training and neurostimulation on sensory-motor skill in esports. Comput. Hum. Behav. 2021:106782. doi: $10.1016 /$ j.chb.2021.106782
Toth, A. J., Ramsbottom, N., Kowal, M., and Campbell, M. J. (2020) Converging evidence supporting the cognitive link between exercise and esport performance: a dual systematic review. Brain Sci. 10:859. doi: 10.3390/brainsci10110859

Wagner, M. G. (2006). "On the scientific relevance of eSports," in International Conference on Internet Computing (Las Vegas, NV), 437-442.

Waris, O., Jaeggi, S. M., Seitz, A. R., Lehtonen, M., Soveri, A., Lukasik, K. M., et al. (2019). Video gaming and working memory: a largescale cross-sectional correlative study. Comput. Hum. Behav. 97, 94-103. doi: 10.1016/j.chb.2019.03.005

Wilms, I. L., Petersen, A., and Vangkilde, S. (2013). Intensive video gaming improves encoding speed to visual short-term memory in young male adults. Acta Psychol. 142, 108-118. doi: 10.1016/j.actpsy.2012.11.003

Yin, K., Zi, Y., Zhuang, W., Gao, Y., Tong, Y., Song, L., et al. (2020). Linking Esports to health risks and benefits: current knowledge and future research needs. $J$. Sport Health Sci. 9:485. doi: 10.1016/j.jshs.2020.04.006

Yuji, H. (1996). Computer games and information-processing skills. Percept. Motor Skills 83, 643-647. doi: 10.2466/pms.1996.83.2.643

Conflict of Interest: The authors declare that the research was conducted in the absence of any commercial or financial relationships that could be construed as a potential conflict of interest.

Copyright (C) 2021 Toth, Frank, Putrino and Campbell. This is an open-access article distributed under the terms of the Creative Commons Attribution License (CC BY). The use, distribution or reproduction in other forums is permitted, provided the original author(s) and the copyright owner(s) are credited and that the original publication in this journal is cited, in accordance with accepted academic practice. No use, distribution or reproduction is permitted which does not comply with these terms. 